\title{
Essais
}

\section{La mort dans la tradition chinoise selon le taoïsme, le bouddhisme et le confucianisme : des conceptions complémentaires}

Death in the Chinese tradition according to Taoism, Buddhism and Confucianism : complementary conceptions

\section{Zhan Wang et Changli Wu}

\section{(2) OpenEdition}

\section{Journals}

Édition électronique

URL : https://journals.openedition.org/essais/8420

DOI : $10.4000 /$ essais. 8420

ISSN : 2276-0970

Éditeur

École doctorale Montaigne Humanités

Édition imprimée

Date de publication : 15 avril 2021

Pagination : 29-37

ISBN : 979-10-97024-09-3

ISSN : $2417-4211$

Référence électronique

Zhan Wang et Changli Wu, « La mort dans la tradition chinoise selon le taoïsme, le bouddhisme et le confucianisme: des conceptions complémentaires », Essais [En ligne], 17 | 2021, mis en ligne le 27 avril 2021, consulté le 18 janvier 2023. URL : http://journals.openedition.org/essais/8420 ; DOI : https://doi.org/10.4000/essais.8420 


\section{La mort dans la tradition chinoise selon le taoïsme, le bouddhisme et le confucianisme : des conceptions complémentaires}

\section{WANG Zhan et Wu Changli}

Dans la tradition chinoise, la mort constitue le point final d'un processus dont la naissance est le point de départ. Contrairement aux religions occidentales monothéistes, le confucianisme, le taoïsme et le bouddhisme chinois n'ont pas pour premier objet la transcendance divine ni même le goût du sacré. C'est pour accéder au bonheur dans le monde que le confucianisme, le taoïsme et le bouddhisme ont œuvré et mené de nombreuses réflexions. Chaque courant part de ses propres croyances pour interroger l'articulation de la mort avec la vie, la famille, la société, la nation, l'univers, etc. En effet, et c'est un des points spécifiques des traditions chinoises, la question de la mort ne cesse d'être interrogée et travaillée, dans la mesure où on considère que c'est une vision claire de la mort qui permettrait de mieux vivre ou tout au moins de s'éloigner du malheur en se rapprochant du bonheur.

\section{La rationalité de l'école confucéenne}

Le confucianisme, éthique sur laquelle la culture chinoise s'est formée, se préoccupe d'abord de la vie et néglige le monde après la mort : à l'égard de la mort, l'école confucéenne demeure dans la rationalité, et nous développons ci-dessous trois points spécifiques de rationalité.

\section{La logique d'alternance du confucianisme}

Le premier point de rationalité : la logique d'alternance. Selon le confucianisme, la mort est un phénomène empirique. Confucius établit sans aucun artifice que "Tout être humain mourra et les morts seront retournés à la terre. ${ }^{1} \mathrm{XuN}_{\mathrm{Zi}}{ }^{2}$, autre penseur de l'école confucéenne, insiste également sur la

1 Mr Dai Shen (Dynastie de Hans Occidentaux), Livret de Rites : discours funèbres (礼记·祭文). Beijing, China Textile \& Apparel Press, 2018, p. 165.

2 Xunzi ou Siun Tseu (313 av. J.-C. - 238 av. J.-C.) est un grand penseur chinois confucianiste. 
mort considérée comme un terme à la vie : « la mort est le point final de l'être humain. ${ }^{3}$ Ces deux propositions sont représentatives d'une part de la pensée confucéenne sur la mort. En effet, si la mort est effectivement un évanouissement, un arrêt, une fin, elle s'inscrit aussi dans un cycle - ce qui permet de se soumettre à une logique de transformation. La vie et la mort sont en réalité deux états en un. La perspective confucéenne développe une logique d'alternance : lorsque quelqu'un nait, un autre meurt. Ceci correspondant d'ailleurs à la typologie dualiste du Yin et du Yang : de la naissance représentée par le Yang à la mort représentée par le Yin, le processus de transformation naturelle est irréversible. Ce fatalisme confucianiste est un stabilisateur. Il est une des méthodes chinoises traditionnelles qui visent à se libérer de l'angoisse de la mort.

\section{La transcendance du confucianisme}

Deuxième point de rationalité : la transcendance dans le confucianisme. Pour le confucianiste, les valeurs morales sont des éléments essentiels pour une vie pleinement réalisée. La morale peut permettre de dépasser dans la pratique la question de la naissance et de la mort, puisque l'individu doit avoir pour seul objectif d'acquérir et de vivre selon des principes moraux qui seront de sa bonne foi. Dans ces conditions, la mort devrait conclure une pleine réalisation individuelle.

La peur de la mort peut aussi entrainer un désir d'immortalité : mais l'école confucéenne renonce à la croyance d'un monde post-mortem. Selon la pensée confucéenne, l'idée que "l'homme aime vivre plus longtemps et déteste mourir trop tôt $»^{4}$ est partagée par tous les hommes. Ceci engendre des projections sur l'au-delà de la mort, projections permettant de rendre le trépas acceptable. La vie est une valeur centrale et la mort est vue comme le malheur absolu. L'immortalité étant impossible, l'école confucéenne se concentre sur la vie mondaine en intégrant la réalité des problèmes sociaux et la quête individuelle de l'au-delà, sans chercher à se préoccuper de l'existence d'un monde post-mortem. La vie sociale est structurée par des règles morales : selon $\mathrm{Zen} \mathrm{Zi}$, disciple de Confucius, "La Voie de notre maître consiste en la loyauté et en l'amour d'autrui comme de soi-même " ${ }^{5}$. La loyauté est ainsi la première règle régissant l'organisation hiérarchique : il s'agit d'une relation verticale qui exige le respect des normes établies. La deuxième est la tolérance qui concerne l'interaction individuelle : c'est une relation horizontale qui exige le respect des coutumes, le dénominateur commun demeurant la «bienveillance». En effet, la bienveillance confucéenne est une sagesse. Confucius souhaite que tous les

3 Xun Zi (Période des Royaumes Combattants), Xun Zi, trad. Yang Liang (Dynastie des Tang), Shanghai, Shanghai Classics Publishing House, 2014, p. 227.

4 Ibid., p. 238.

5 Confucius, Les entretiens de Confucius, Anne Cheng (trad.), Paris, Éditions du Seuil, 1981, p. 17. 
hommes s'aiment les uns les autres et évitent la rivalité qui les blessera tous. Pour parvenir à ce but - l'accession à une vie harmonieuse -, tous les individus doivent lutter contre leurs propres envies tout en respectant les normes sociales. La sagesse confucéenne développe une conceptualisation transversale de la mort et se met en quête d'un principe : celui de coexistence collective. Elle a pour objectif de déplacer la préoccupation individuelle pour le monde post-mortem vers le monde matériel, de façon à éloigner la souffrance qu'engendre la méconnaissance ou l'incertitude relatives au monde post-mortem.

\section{L'esprit de sacrifice dans le confucianisme}

Dans le troisième point de rationalité confucéenne, nous abordons le sacrifice pour une cause juste. En effet, la mort d'un individu doit s'évaluer selon les valeurs comme la bienveillance, la justice, les rites, la sagesse et la foi. ${ }^{6} \mathrm{D}$ 'après Confucius, le meilleur moyen de transcender la mort est de développer la bienveillance. Ceci relève de la responsabilité d'un individu qui doit établir un objectif personnel pour sa vie. La bienveillance est une manière de se réaliser et de survivre au-delà de la mort. Pour cela on peut aller jusqu'à se sacrifier pour une cause juste. Confucius déclare : "Celui qui le matin a compris la Voie, le soir peut mourir content. $»^{7}$ Mencius $^{8}$, une autre figure de l'école confucéenne, insiste sur la justesse d'esprit aussi bien que la justice : "Le vivre, c'est ce que je veux ; la justice, c'est aussi ce que je veux. Je me soumets à l'abandon de ma vie pour la justice lorsque je suis devant un seul choix. " ${ }^{9}$ Confucius et Mencius imaginent de pouvoir se sacrifier en faveur de la morale, ceci débouchant sur l'accomplissement ou la réalisation personnelle. Pour Confucius, l'idéal de vertu subsume tous les autres : « Un gentilhomme d'idéal, un homme pleinement humain ne cherche jamais à sauver sa vie aux dépens de la vertu d'humanité. Il est des circonstances où il sacrifie sa vie, pour que s'accomplisse cette vertu. ${ }^{10}$ Cette valeur est pour Confucius une solution visant à dépasser la peur de la mort : se perfectionner par la morale et se sacrifier pour la cause juste. De cette façon, l'individu atteint sa finalité ultime grâce à des comportements altruistes, tout en négligeant le départ physique du corps.

Confucius s'est rendu compte que dans la pratique, il valait mieux ne pas se soumettre au fatalisme vis-à-vis de la mort. Il faut donc opter pour un triptyque individuel (« la vertu, les mérites et la doctrine. Une fois ces trois points

6 Lesegretain Claire, Le Confucianisme [EB/OL], 2011. https://www.la-croix.com/Archives/201101-08/Le-Confucianisme.-Le-confucianisme-_NP_-2011-01-08-393765.

7 Confucius, Les entretiens de Confucius, op. cit., p. 16.

8 Mencius (380 av. J.-C. - 289 av. J.-C.) est un penseur chinois confucéen.

9 Mengzi, Mengzi, Nanchang, $21^{\text {st }}$ Century Press, 2014, p. 288.

10 Confucius, Les entretiens de Confucius, op. cit., p. 78. 
établis, la réputation individuelle perdurera et sera immortalisée $»^{11}$ ) afin que les individus puissent transcender la mort. Le triptyque préconisé par Confucius s'offre comme un principe, orientant les comportements individuels, qui permet de faire face au fatalisme. De plus, l'école confucéenne met l'accent sur la valeur de l'individu en immortalisant son souvenir : l'identification des mérites permet d'inscrire les noms de famille dans l'histoire et d'honorer ainsi les ancêtres.

La transformation des préoccupations liées à l'au-delà en préoccupations terrestres relève d'une rationalité stratégique : d'une part, la conduite de l'existence individuelle ne repose plus sur la peur instinctive de la mort, mais sur une finalité idéologique fondée sur l'alternance, le sacrifice et la transcendance. D'autre part, elle procède d'une fonction à la fois idéale et utilitaire, en vue du fonctionnement social.

\section{Le concept naturel du taoïsme}

Contrairement au confucianisme, le concept taoïste de la mort est moins concerné par la valeur de la vie et accorde une grande attention au sens de la mort. Nous parlerons dans ce qui suit de trois points : la Loi de la nature, la libération pour transcender, et la quête d'éternité.

\section{La loi de la nature taoïste}

Pour l'école taoïste ${ }^{12}$, l'homme doit vivre en fonction de la loi terrestre. La terre donne la vie à tous les êtres selon des états climatiques. Le ciel qui tourne au-dessus de nous selon le Tao ordonne le climat. Le Tao atteint sa finalité en suivant la nature. La loi taö̈ste s'impose comme un règlement à caractère naturel. Rien n'agit au-delà de la nature. Le Tao est un état indépendant. ${ }^{13}$ Le monde n'est constitué de rien et n'interagit pas dans les rapports interpersonnels.

Cette philosophie peut aussi s'appliquer dès lors qu'on aborde la question de la mort. Les individus doivent aimer la vie et aspirer à l'immortalité, mais sans cupidité. Car la cupidité engendre immanquablement la faute. Pour

11 Zuo Qiuming, Chunqiu-Zuozhuan:Xianggong.le, $24^{\mathrm{e}}$ année (左氏春秋 : 裹公·裹公二十四年) [EB/OL]. https://so.gushiwen.org/guwen/bookv_2955.aspx.

12 JJL Duyvendak, Tao Te King de Lao Tseu, Le Livre de la Voie et de la Vertu [EB/OL]. https:// www.taichi-kungfu.fr/dossiers-chine-culture/dossier-philosophie-chinoise/taoisme/lao-tseutao-te-king-le-livre-de-la-voie-et-de-la-vertu-duyvendak-dao-de-jing-traduction/\#Lao-TseuTao-Te-King-Duyvendak-Laozi-Dao-De-Jing-1.

13 Luo Zhongshu, Pensée de Chong Xuan : une étude de la méthode et de l'épistémologie de Cheng Xuanying à Chong Xuan (重玄之思: 成玄英的重玄方法和认识论研究), Chengdu, Press de Bashu, 2010, p. 20. 
Zhuang $\mathrm{Zi}^{14}$, autre figure de l'école taoïste, les hommes n'ont ni corps ni énergie (souffle) avant d'être nés. C'est sous l'influence du Tao que l'on constate une évolution de la source vivante vers une forme corporelle et enfin un être humain. La mort et la vie sont le changement d'une période à une autre tout comme les quatre saisons. Il s'agit d'un mouvement spontané en fonction des lois de la nature. De la vie à la mort ou de la mort à la vie, ce n'est pas un sens unique, mais une voie (Tao) double. Ainsi, l'école taoïste met en valeur la notion de repos. À la différence du triptyque confucéen, l'école taoïste repose sur une attitude naturelle : la mort ou la vie sont arrangées par le ciel, et la mort est le moment du repos.

\section{La libération pour la transcendance}

La rapidité de la vie est génératrice d'angoisse. L'homme est souvent confronté à une menace naturelle ou à un risque social sans issue, comme le rappelle Zhuang $\mathrm{Zi}$ : "On est né d'une peur qui tient jusqu’à la mort. ${ }^{15}$ Mais alors pourquoi tient-on tellement à cette vie pleine de douleur ? Les taoïstes prennent la mort comme repos et considèrent que l'avènement de la mort est précisément le meilleur moment pour se reposer. Le concept taoïste de "prendre la mort comme un intérêt » est complètement une attitude naturaliste.

C'est une vision pessimiste qui amène les individus à se précipiter vers une issue qu'offre la mort en termes de libération. La libération permet de se dégager des contraintes mondaines sans nécessairement rechercher la vérité ou le sens de la vie. C'est ce que préconise l'école taoïste : l'apprentissage du savoir vivre. Celui qui comprend les changements naturels sans perturbation est qualifié par Zhuang $\mathrm{Zi}$ de "Cai-Quan ${ }^{16}$. Cai-Quan est celui qui vit dans le bonheur et meurt dans la tranquillité. Il peut aller au-delà de la mort. L'objectif est non pas de susciter le désir des hommes en vue de chasser la mort, mais plutôt de leur permettre d'accéder à un esprit calme et tranquille. Il s'agit de se hisser vers le détachement spirituel notamment à travers l'éloignement de préoccupations engendrées par un monde complexe et plein de mutations imprévisibles.

\section{La recherche d'éternité}

L'accès à la vie éternelle est le souhait de tous. D'après l'école taoïste, la mort et la vie relèvent d'une loi universelle de laquelle aucun individu ne peut s'extraire. "La mort d'un individu est comme la cendre provenant d'un foyer

14 Zhuangzi ou Tchouang-Tseu (369 av. J.-C. - 286 av. J.-C.) est un penseur chinois du taoïsme.

15 Zhuangzi, Zhuangzi, Nanchang, $21^{\text {st }}$ Century Press, 2014, p. 167.

16 Ibid., p. 47. 
éteint. Tout individu n'a qu'une vie et ne peut plus se régénérer lorsqu'il est parti. $"{ }^{17}$ Lécole taoïste propose cependant des techniques pour échapper à l'enchevêtrement du monde mortel : régimes diététiques, gymnastique, etc., mais également utilisation d'élixirs divers. Pour cultiver son énergie vitale on peut aussi consommer des " pilules d'immortalité ». Ces pilules devaient être fabriquées dans des espaces précis, dans des fourneaux spécifiques et des marmites exclusivement en terre cuite, contenant par exemple du sel gemme et des poils de chèvre. Les pilules étaient fabriquées à partir de huit substances, parmi lesquelles du cinabre, du réalgar, de l'orpiment et du sulfate de zinc ${ }^{18}$.

Une autre façon d'être immortel consiste à ne pas considérer la mort comme un décès : le corps est mort, mais la vie demeure éternellement. Dans le monde physique, la vie ou la mort sont des états temporaires dans une infinité de changements. Si l'on veut se débarrasser de la mort en quelque sorte, se libérer des souffrances et des douleurs, il est nécessaire de se concentrer sur le monde spirituel : nous pouvons ainsi négliger ou oublier la joie ou le bonheur. C'est une expérience psychologique qui ressemble à la méditation zen : une concentration sur soi-même pour dépasser le monde matériel et par la suite se sublimer dans un monde éternel.

La philosophie taoïste est donc une position métaphysique cherchant à échapper à la dialectique de la vie et de la mort.

\section{Les concepts de réincarnation du bouddhisme}

Troisième étape de notre parcours, le bouddhisme. Le bouddhisme nie souvent le monde réel et accorde plus d'attention au monde illusoire, qui a un impact négatif sur la vie laïque réelle. Dans cette partie, nous abordons de même que précédemment trois points : la délivrance des souffrances, la réincarnation et le nirvana.

\section{La délivrance des souffrances}

Lorsque le "petit véhicule 19 " bouddhiste est introduit en Chine avec sa spécificité de viser à la libération de la peur individuelle, on constate que celui-ci s'adapte assez bien avec le "grand véhicule ${ }^{20}$ dont l'objectif consiste à aider les hommes à se débarrasser de la panique face à la mort. Pour le bouddhiste chinois, la quotidienneté, la vieillesse, la maladie, la mort, la haine, la

17 Taipingjing, Shanghai, Classics Publishing House, 2013, p. 171.

18 "La pilule de l'immortalité ", Revue d'Histoire de la Pharmacie, 145, 1955, p. 124-125.

19 Petit véhicule : traduction de l'expression sanscrite " hīnayāna »; désigne les école anciennes du bouddhisme.

20 Grand véhicule : traduction d'un terme sanscrit " mahāyāna ". 
séparation et la perte produisent des souffrances. Se délivrer de la souffrance signifie la libération définitive de l'âme individuelle. Le processus d'élimination passe par l'appréhension, la pratique et la méditation.

Le bonheur et la douleur accompagnent toute la vie et produisent à la fois un désir de longévité ainsi que l'angoisse de la mort. Pour faire face aux douleurs engendrées par la concurrence, le bouddhisme souligne qu'il n'est pas nécessaire pour l'homme de chérir sa vie ni de la prolonger, car le malheur est constant et le bonheur inconstant. Le bouddhisme a donc exagéré les souffrances de la vie afin de dissiper les craintes liées à la mort et les pathologies sociales de l'individu. C'est une sagesse qui tend à montrer que la vie n'est pas aimable.

\section{La réincarnation}

Dans cette perspective, le bouddhisme s'attache au principe de la réincarnation : un être humain peut vivre de nombreux cycles de vie, la mort n'étant qu'un état transitoire au sein de ce cycle de vie indépendant de sa volonté. La vie est un destin auquel nul ne peut échapper : la réincarnation est un moyen d'obtenir l'éternité par-delà la mort ou la vie. Selon les principes bouddhistes, la réincarnation se répartit en six univers : celui du dieu, celui des divins, celui de l'humain, celui de l'animal, celui du fantôme affamé et celui des enfers ${ }^{21}$. Les trois derniers sont des espaces maléfiques alors que les trois premiers sont des espaces bénéfiques. La vie s'efface et revient en une continuité circulaire : le cycle consiste en une succession de moments terrestres, dont la qualité est liée au vécu précédent. Cette successivité par paliers doit apporter la sagesse au fur et à mesure des incarnations, c'est ainsi que l'individu peut aboutir à l'univers de Bouddha, l'univers des dieux où toute personne est éternelle.

\section{Le nirvana}

Pour parvenir à l'univers de Bouddha, on imagine le principe du nirvana. Puisque le mot nirvana signifie "éteindre ", il s'agit de mourir heureux sans désir ni douleur, c'est-à-dire en éteignant en soi le désir et la douleur. Lorsque la " conscience " quitte le corps, elle accède à une nouvelle forme de vie, soit dans un autre individu, soit dans un animal ou encore un fantôme, jusqu'à l'accès à l'univers du Bouddha ${ }^{22}$. Comme le désir est destructeur et aveugle, on doit l'éloigner de soi. Pour cela, il faut respecter certaines règles : d'un côté, il faut ne pas travailler, ne pas s'enrichir et ne pas se soumettre à la sexualité ; d'un autre côté, il convient de traiter les autres avec bonté et pratiquer la méditation. Ces pratiques selon les bouddhistes permettent de sortir

21 Hugues St-Pierre, Conception et expériences de la mort dans le bouddhisme [EB/OL], 2017. http://montagnedesdieux.com/conception-et-experiences-de-la-mort-dans-le-bouddhisme/.

22 Le Nirvana [EB/OL]. https://www.bouddhiste.net/initiation-au-bouddhisme/le-nirvana/. 
du cycle des réincarnations dans la mesure où elles amènent à une meilleure compréhension de soi, de la destinée humaine, tout en renforçant l'esprit pour éteindre le désir.

Après avoir montré les trois principales propositions chinoises pour domestiquer la mort (l'immortalité sociale du confucianisme, le naturalisme philosophique du taoïsme, et l'abnégation du bouddhisme), nous nous proposons dans ces quelques lignes de conclusion de rendre compte de la plasticité du sentiment religieux chinois, en tant qu'il est capable de faire la synthèse de ces trois visions si différentes de la mort.

La sagesse confucéenne consiste à rationaliser la fatalité de la vie humaine par l'alternance entre la vie et la mort. Cette sagesse, sans nier la transcendance, se préoccupe d'abord de la réalité sociétale avant toute considération autour du monde post-mortem. Dans la pratique, l'exigence porte sur les valeurs, soit le sacrifice pour une cause de justice.

La stratégie taoïste consiste à respecter la loi de la nature. La mort ou la vie sont arrangées par le ciel et le repos vient lorsqu'on meurt. On transcende donc la vie et la mort que cela soit heureux ou douloureux. La transcendance est concrétisée par l'amélioration d'énergie et la fabrication de pilules d'immortalité.

La technique bouddhiste consiste en la libération de l'âme pour ôter la douleur en mettant en avant la notion de réincarnation pour atteindre l'univers des dieux, soit l'éternité. Dans la pratique, on doit maintenir le nirvana, c'est-à-dire notamment éteindre le désir qui est destructeur. Pour cela, il faut ne pas travailler ni s'enrichir ni se soumettre à aucune forme de sexualité, tout en traitant les autres avec bonté et en pratiquant la méditation.

Les conceptions chinoises sur la mort diffèrent de celles du christianisme dès leurs principes théoriques. Contrairement à l'importance donnée à l'autolibération dans les conceptions traditionnelles chinoises sur la mort, le christianisme est marqué profondément par le péché originel et l'individu a peu ou prou (selon les orientations catholiques ou protestantes) besoin de l'intervention divine pour son rachat. C'est en croyant en Dieu et en utilisant toute la vie pour se repentir du péché, que l'on peut espérer sauver son âme. Le confucianisme, le taoïsme et le bouddhisme se concentrent sur l'être humain, tandis que le christianisme est une religion qui considère premièrement Dieu. Le christianisme relève de la transcendance externe reposant sur le pouvoir de la croyance en Dieu, alors que les conceptions traditionnelles chinoises manifestent davantage la transcendance interne du soi, bien qu'elles contribuent également à des forces extérieures : par exemple, le sacrifice pour la réussite de la cause juste pour les confucianistes, la " pilule d'immortalité » dans le taoïsme, la croyance en Guanyin dans le bouddhisme, etc. La religion chinoise met généralement l'accent sur l'amélioration de soi. 
Par conséquent, nous devrions adopter une attitude critique et prendre ce qu'il y a de plus précieux dans le concept traditionnel de vie et de mort tout en éliminant le mauvais, afin de trouver une méthode efficace pour résoudre la crise moderne de la survie. Dans le même temps, tout en faisant appel à la sagesse de la vie et de la mort dans le concept traditionnel de la vie et de la mort, nous devons également absorber activement la culture occidentale avancée, pour que le développement de la nation chinoise soit synchronisé avec le monde et que la culture chinoise joue un rôle important dans le développement de la culture mondiale.

\section{Wang Zhan et Wu Changli \\ Université de Wuhan wangz13@aliyun.com}

\section{Résumé}

La mort est ici examinée du point de vue de l'éthique traditionnelle. Nous présentons d'abord de manière générale les différentes conceptions de la mort dans la tradition chinoise, selon le confucianisme, le taoïsme et le bouddhisme ainsi que leurs origines en lien avec l'organisation sociale. Dans le cadre de cet article, nous nous bornons à montrer de quelles manières, avec quels outils théoriques et pratiques ces traditions tentent de répondre à l'angoisse de la mort dans la Chine ancienne et moderne.

\section{Mots-clés}

Concept de la mort, confucianisme, taoïsme, bouddhisme.

\section{Abstract}

Death is examined here from the point of view of traditional ethics. We first present in a general way the different conceptions of death in the Chinese tradition, according to Confucianism, Taoism and Buddhism as well as their origins in connection with social organization. Within the framework of this communication, we limit ourselves to showing in which ways, with which theoretical and practical tools these traditions try to respond to the anxiety of death in ancient and modern China.

\section{Keywords}

Death concept, Confucianism, Taoism, Buddhism. 